\title{
Monitoring of adenovirus serotypes in environmental samples by combined PCR and melting point analyses
}

\author{
Nils Marten Hartmann*, Melanie Dartscht, Regine Szewzyk and Hans-Christoph Selinka
}

\begin{abstract}
Background: Human adenoviruses are promising candidates for addressing health risks associated with enteric viruses in environmental waters. Relatively harmless but common, these DNA viruses persist within the population and are generally considered extremely stable, remaining infectious in water for long periods of time. Group-specific or single species detection of human adenoviruses in environmental samples is usually based on polymerase chain reaction assays. Simultaneous identification of specific species or serotypes needs additional processing. Here we present a simple molecular approach for the monitoring of serotypic diversity in the human adenovirus populations in contaminated water sites.
\end{abstract}

Methods: Diversity patterns of human adenoviruses in environmental samples, collected in an outdoor artificial stream and pond simulation system, were analyzed using a closed tube polymerase chain reaction method with subsequent melting point analysis.

Results: Human adenovirus serotype 41 was the most prominent adenovirus serotype detected in environmental water samples, but melting point analyses indicated the presence of additional adenovirus serotypes.

Conclusions: Based on investigations with spiked and environmental samples, a combination of qPCR and melting point analysis was shown to identify adenovirus serotypes in sewage contaminated water.

Keywords: Adenovirus, Environment, Melting point analysis, aPCR, Sewage, Viral indicator, Virus stability

\section{Background}

Contamination of surface waters by sewage can be a serious hazard to the environment and a potential risk to human health [1,2]. Microbes, viruses and chemical substances may impact animal and plant reproduction as well as egg and larval development [3-5]. Introduction of high nutrient concentrations can induce eutrophication. Furthermore, microbial pollution can affect the stability and composition of microbial communities or ecosystems, depending on the dimension of contamination and the tolerance of the water system $[1,6,7]$. Moreover, infections by sewage-derived waterborne pathogens may affect the health of both animals and humans alike $[8,9]$. Studies indicate that viruses often persist for several months in contaminated waters and

\footnotetext{
* Correspondence: nils.hartmann@uba.de

German Federal Environment Agency (UBA, Umweltbundesamt), Division ॥ 1.4 Microbiological Risks, Corrensplatz 1, 14195 Berlin, Germany
}

are detected far longer than indicator bacteria [10-12]. In addition, detection of infectious viruses is more complicated than detection of indicator bacteria, like Escherichia coli and intestinal enterococci, both used to detect fecal contaminations in water, and few viral infectious units may cause disease. For instance, 1-10 infectious particles of rotavirus can cause disease in animal or human models, whereas, at similar levels of exposure, the risk of infection with enteropathogenic bacteria is about 10-10.000 times lower than for viruses $[12,13]$. Reliable indicators for contaminations with human viruses would be important tools to improve and monitor the quality of water bodies, such as bathing waters or drinking water reservoirs. However, selection of a viral indicator is difficult, since human viruses vary in their abundance, virulence, environmental resistance, transport behavior, epidemiological significance, ecology and also in their methods of detection $[12,14,15]$. The Contaminant

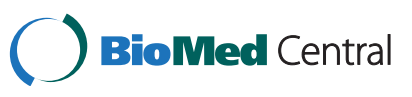


Candidate List (CCL), published by the United States Environmental Protection Agency (EPA), lists priority candidates for research in order to facilitate necessary decisions for further regulations. Among the listed viruses are also human adenoviruses [16]. To achieve a higher level of water quality, the World Health Organization (WHO) suggests to perform risk assessments based on the monitoring of one or more representatives of the large group of enteric viruses, e.g. enteroviruses, astroviruses, enteric adenoviruses, orthoreoviruses, rotaviruses, caliciviruses, hepatitis A or E viruses, in addition to bacterial indicators [17]. Viruses that are more readily detected by current available methods are recommended for routine monitoring, which include the members of the enterovirus, adenovirus and orthoreovirus groups. All of these virus taxa comprise many species, serotypes and genotypes, some of them differing strongly with regard to stability and tenacity.

Detection of viruses in environmental samples is usually based on polymerase chain reaction (PCR) and plaque assays. While the latter are time-consuming and intricate, numbers of infectious viruses may be overestimated by PCR based methods as only nucleic acids are detected. PCR based methods may also be subject to inhibitory effects, but are sensitive in general and results can be obtained within few hours [18]. In addition, virus detection by PCR methods can be used for specific monitoring of multiple virus types simultaneously, while plaque assays may completely fail to detect certain virus types, when fast growing competitive strains are present $[19,20]$. For example human adenovirus [hAdV] serotype 2 is released twice as fast by infected HEK293 cells as the slow growing fecal adenoviruses and may completely lyse a cell layer before human adenovirus serotype 41 will show any visible effect, especially when different amounts of these viruses are present in the same sample [21]. Moreover, adenovirus cultivation is clearly influenced by the cell line used. In particular, using plaque systems, detection of hAdV41 is critical in the presence of species $\mathrm{C}$ adenovirus serotypes, while simultaneous detection and quantification of both serotypes is no problem using multiplex quantitative real time PCR (qPCR) [22,23]. However, infectivity cannot be demonstrated by direct PCR measurements alone, but by application of cell culture-based methods, like plaque assays or integrated cell culture PCR (ICC/qPCR) [24]. As long as no better approaches are available and affordable, monitoring should ideally be performed by combining PCR and cell culture-based assays [22].

Most methods for virus detection and typing were designed for testing of clinical samples, and only few of them can be applied to environmental samples without modifications and additional processing. While many virus methods are either suitable for detection, quantification or typing, melting point analysis (MPA) is an inexpensive and fast alternative, which can be directly connected to qPCR amplification without further processing and additional equipment. Since even single nucleotide exchanges lead to shifts in the melting points of specific sequences, the technique is often used for mutation scanning in clinical samples $[25,26]$. However, so far, this method has not been applied to environmental samples for characterization of viruses. Here, we demonstrate the use of PCR product melting points for heterogeneity analyses and its suitability for direct identification of human adenovirus serotypes in sewagecontaminated samples.

\section{Results}

Serotypes of all known human adenovirus species, except for the single serotype in species G, were tested for their stability in processed water in order to investigate their potential use as viral indicators. Human adenovirus serotypes 1, 2 and 5 (species C), 4 (species E), 11 (species B), 22 (species D), 31 (species A) and 41 (species F) were investigated. Concentrations of all examined serotypes did not decrease for more than $1.5 \log _{10}$ steps at $6( \pm 2)^{\circ} \mathrm{C}$ within eight weeks (Figure 1). While some viruses decreased stronger during the early incubation phase (serotypes 1, 2, 4 and 41), others showed a higher logarithmic decrease during later times (serotypes 5, 11 and 31), whereas hAdV22 decreased constantly over time. The mean reduction in copy numbers of all tested adenoviruses was in the range of about $0.5 \log$ in 32 days and about $1.0 \mathrm{log}$ in 64 days. hAdV41 displayed a decrease in copy numbers of $1.0 \log _{10}$ unit at $6^{\circ} \mathrm{C}$ and $3.0 \log _{10}$ units at room temperature (Figure 2). Regarding virus stability, the results demonstrate, that all of the tested serotypes would potentially qualify for biodiversity analyses or could

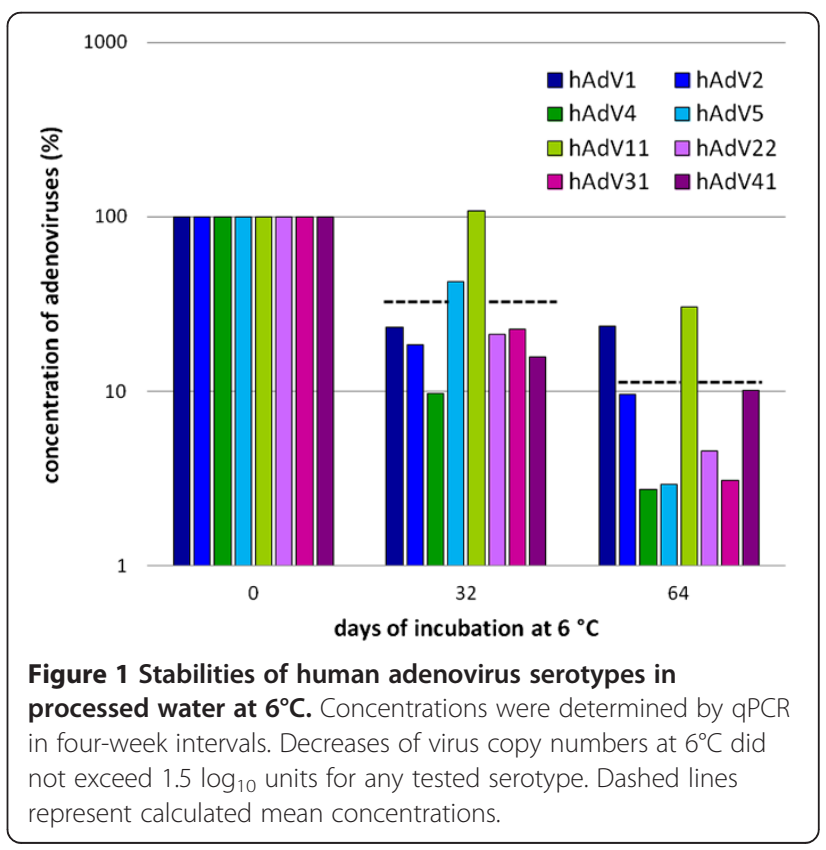




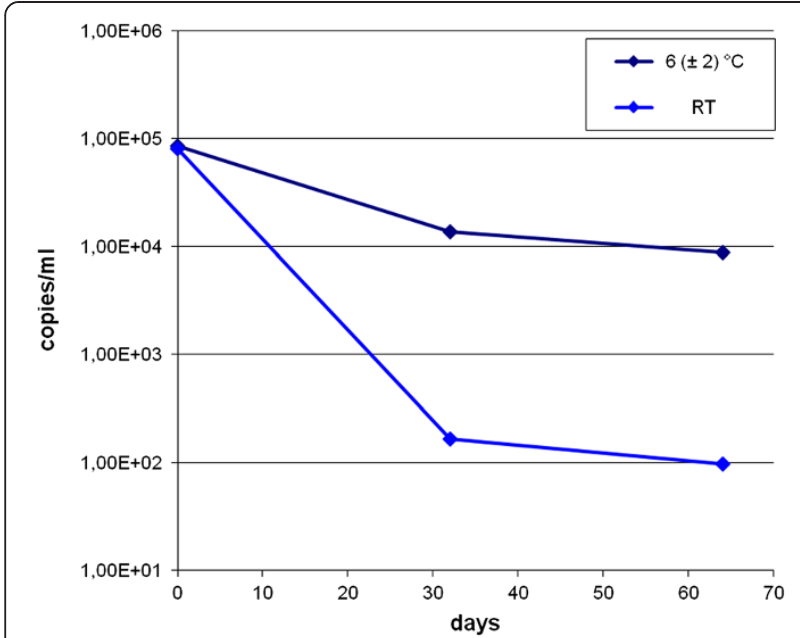

Figure 2 Decrease of human adenovirus serotype 41 in processed water at distinct temperatures over time. The decrease of virus copy numbers at room temperature exceeded the decrease at $6^{\circ} \mathrm{C}$ by about $2 \log _{10}$ units.

be used as indicators for contaminations with human viruses derived from municipal sewage.

For melting point analyses following qPCR amplifications, the primer pair AdF/AdR, designed by Hernroth et al. (2002), was used [27]. Distinct melting points $\left(\mathrm{T}_{\mathrm{m}}\right)$ were identified for several adenovirus serotypes. Melting points and relative shifts largely confirmed theoretical calculations using sequences provided by NCBI, amended by the primer sequences, and the OligoCalc program [28]. Since degenerated primers were used, melting points of serotype-specific amplification products are listed in temperature ranges (Table 1 ).

Typical melting curves for several human adenovirus serotypes are shown in Figure 3. Peak positions of the type-specific adenovirus melting curves were mostly stable and standard deviations were generally low (Table 1). Serotypes hAdV2, hAdV22 and hAdV41 showed overlying melting curves with maxima too close for discrimination in any serotype mixed sample. Similar results were obtained for hAdV1 and hAdV5, belonging to the same adenovirus species as hAdV2 (data not shown). However, several adenovirus serotypes displayed clear distinguishable peak positions.

In a second set of experiments, melting point analysis was applied to environmental water samples. These samples derived from tenacity experiments performed in an artificial stream and pond simulation system with sewagecontaminated water containing human adenoviruses. Samples were drawn in the beginning and at the end of experiments, spanning a time interval of several weeks. Conspicuous in some graphs were pronounced shoulders or multiple peaks, putatively indicating serotype heterogeneity (Figure 4). In several cases peak intensities
Table 1 Comparison of calculated and measured melting points

\begin{tabular}{lccc}
\hline Serotype & Species & Calculated $\mathbf{T}_{\mathbf{m}}$ & Measured $\mathbf{T}_{\mathbf{m}}$ \\
\hline hAdV11 & B & 81.03 to $81.82^{\circ} \mathrm{C}$ & $81,10 \pm 0.19^{\circ} \mathrm{C}$ \\
hAdV4 & E & 81.37 to $82.14^{\circ} \mathrm{C}$ & $81,69 \pm 0.30^{\circ} \mathrm{C}$ \\
hAdV31 & A & 81.66 to $82.41^{\circ} \mathrm{C}$ & $82,11 \pm 0.23^{\circ} \mathrm{C}$ \\
hAdV22 & D & 83.08 to $83.32^{\circ} \mathrm{C}$ & $83,28 \pm 0.19^{\circ} \mathrm{C}$ \\
hAdV41 & F & 83.47 to $84.16^{\circ} \mathrm{C}$ & $83,28 \pm 0.27^{\circ} \mathrm{C}$ \\
hAdV40 & F & 83.57 to $84.25^{\circ} \mathrm{C}$ & $80,38 \pm 1.23^{\circ} \mathrm{C}$ \\
hAdV1 & C & 83.57 to $84.25^{\circ} \mathrm{C}$ & $83,78 \pm 0.06^{\circ} \mathrm{C}$ \\
hAdV2 & C & 83.57 to $84.25^{\circ} \mathrm{C}$ & $83,26 \pm 0.48^{\circ} \mathrm{C}$ \\
hAdV5 & C & 83.57 to $84.25^{\circ} \mathrm{C}$ & $83,86 \pm 0.19^{\circ} \mathrm{C}$ \\
\hline
\end{tabular}

declined dramatically over time, as exemplarily shown for experiment $\mathrm{V}$ (green lines). The peak positioned at about $84^{\circ} \mathrm{C}$, most often the highest or main peak, was always overlapping with the reference peak (fecal adenovirus serotype 41). These results were also confirmed by restriction fragment analyses, and, for some samples (exp. II), by direct sequencing (data not shown). In contrast, high temperature peaks (peaks exceeding $85^{\circ} \mathrm{C}$ ) could not be assigned to any reference virus used for melting point analyses in our experiments. Since the applied primer system was originally designed for group-specific adenovirus detection, primers contained degenerated base positions. Therefore, the specificity of the primers was analyzed for all known human adenovirus serotypes (56 serotypes) and several zoopathogenic mastadenovirus species. However, none of the predicted melting points surpassed $85^{\circ} \mathrm{C}$.

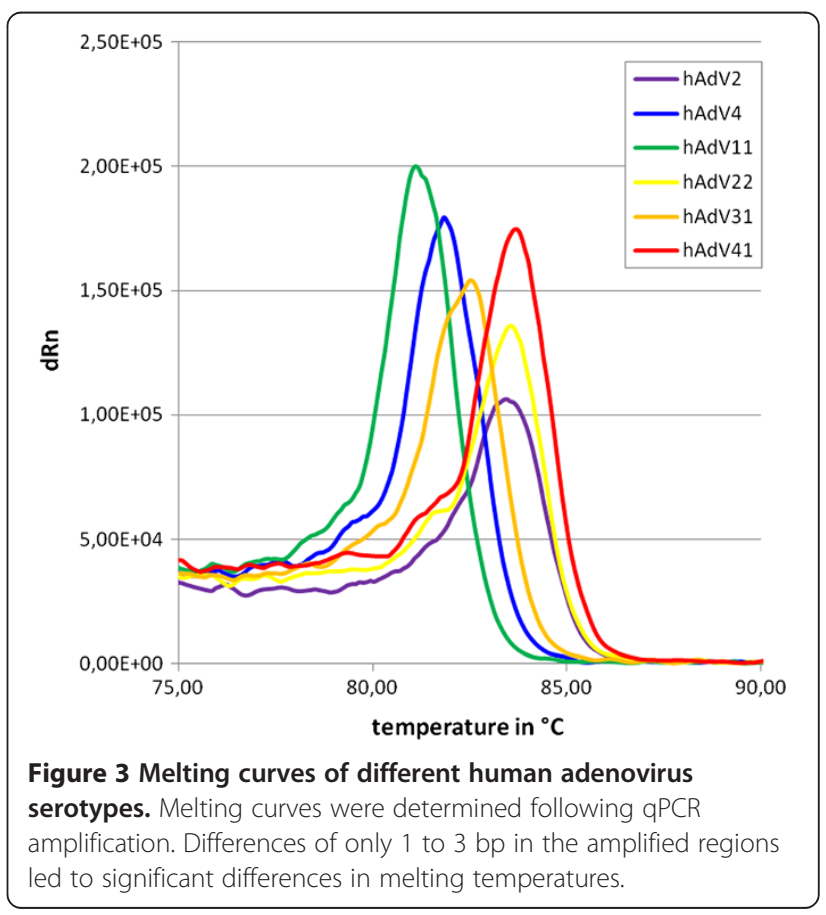




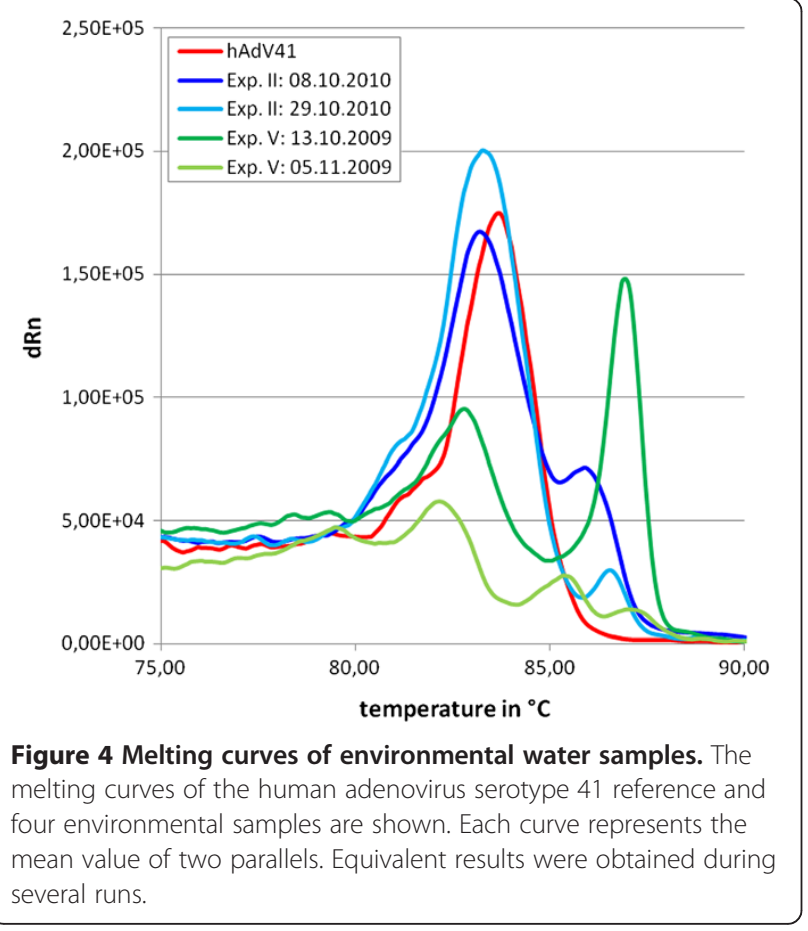

Table 2 lists the number of nucleotide differences of primer and target sequences. Only the reference serotypes as well as hAdV3 (species B, maximal nucleotide mismatch number), hAdV52 (species G) and certain animal serotypes are displayed. The number of mismatches was identical for all serotypes of the species $C$ and $D$, respectively. hAdVE and hAdVG consist of only one known serotype (serotype 4 and serotype 52, respectively). Significant nucleotide matches with non-adenovirus sequences were not predicted by blastn, and highest alignments were always below $90 \%$. The primer pair AdF/AdR is usually used in combination with the TaqMan probe ACDEF for groupspecific adenovirus qPCR [27]. Therefore, both the differences in probe and target sequences were determined (Table 2). Although primer alignments showed higher mismatch numbers for most investigated animal adenoviruses than for human adenoviruses, most of the mismatches concerning the reverse primer $\mathrm{AdR}$ are located at the 5'-prime end, the end which is not extended during polymerization. However, during $\mathrm{qPCR}$ the number of mismatches will most likely prevent probe association in most cases. In our melting point analyses SYBR green was used and detection of non-human adenoviruses cannot be excluded. Therefore, other primer systems were investigated for their specificity and applicability in melting point analyses, namely the primer pairs Hex1deg/Hex2deg and neHex3deg/neHex4deg (Figure 5). These primer pairs were originally designed for nested PCR based detections of human adenoviruses [29]. While 69 base pair (bp) amplicons were produced using the AdF/AdR primer pair,
Table 2 Nucleotide mismatches between primer, probe and target sequences

\begin{tabular}{lccc}
\hline Serotype or species $^{\mathbf{a}}$ & Fw $^{\text {primer }}{ }^{\mathbf{b}}$ & Rv $_{\text {primer }} \mathbf{b}^{\mathbf{b}}$ & Probe $^{\mathbf{b}}$ \\
\hline hAdV31 [A] & 0 & 0 & 3 \\
hAdV3 [B] & 0 & 0 & 4 \\
hAdV11 [B] & 0 & 0 & 3 \\
hAdV2 [C] & 0 & 0 & 1 \\
hAdV22 [D] & 0 & 0 & 0 \\
hAdV4 [E] & 0 & 0 & 2 \\
hAdV40 [F] & 0 & 0 & 0 \\
hAdV41 [F] & 0 & 0 & 1 \\
hAdV52 [G] & 0 & 0 & 2 \\
bAdVA & 1 & 3 & 6 \\
cAdV1 & 2 & 3 & 7 \\
cAdV2 & 2 & 3 & 7 \\
fAdV & 0 & 0 & 2 \\
mAdV2 & 1 & 2 & 5 \\
oAdVA & 0 & 3 & 7 \\
pAdVC & 1 & 3 & 3 \\
\hline
\end{tabular}

${ }^{a}$ Human adenovirus species are listed in brackets. For abbreviations and accession numbers, see Methods section.

${ }^{\mathrm{b}}$ Forward primer, reverse primer and probe, designed according to Hernroth et al. (2002).

173 bp amplicons were produced using the neHex3deg/ neHex4deg primer pair and 301 bp amplicons by using the Hex1deg/Hex2deg primer pair [27,29]. Sequence differences between related serotypes where expected to be higher in longer amplicon sequences and thus expected to allow better melting point discriminations. Analysis of primer specificities using the neHex3deg/neHex4deg primer pair instead of the AdF/AdR primer pair revealed lower affinities for animal adenovirus sequences than for human adenovirus sequences. Differences of animal and human adenovirus sequences using the Hex1deg/Hex2deg primer pair were comparable to differences using the $\mathrm{AdF} / \mathrm{AdR}$ primer pair, with generally slightly reduced specificities using the latter primer pair. QPCR analyses yielded comparable results for all three primer pairs, using the pAdV41 standard as target. Although higher melting temperatures were achieved with longer primer sequences, amplification efficiencies for the different serotypes were best using the AdF/AdR primer system (data not shown). The choice of appropriate primer pairs is therefore critical for the monitoring of virus serotypes in environmental water samples.

\section{Discussion}

Temperature is an important trigger to abiotic virus destruction. Therefore stability analyses with selected adenovirus serotypes were performed. The majority of the examined serotypes displayed similar innate stabilities at 


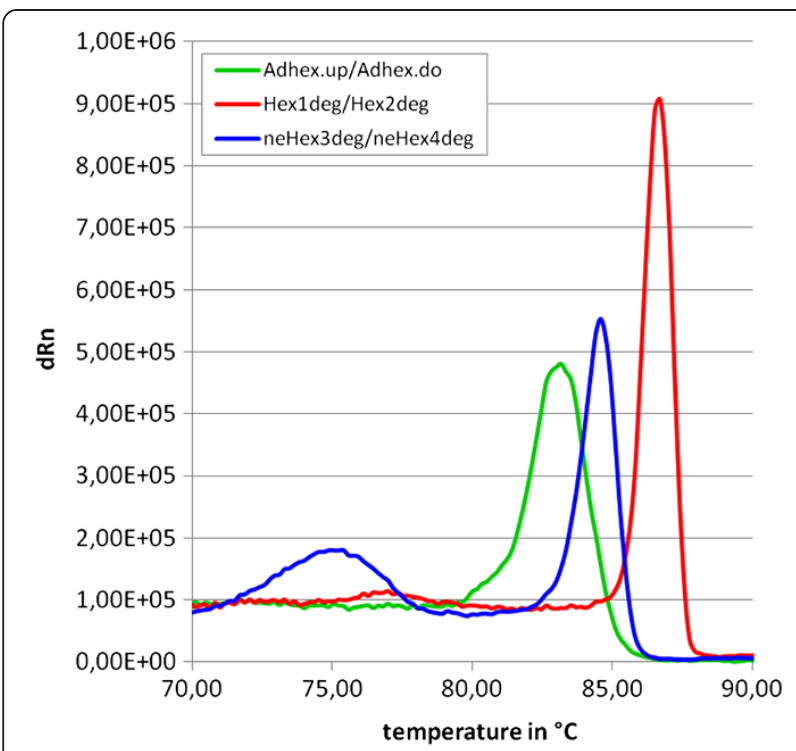

Figure 5 Melting curves of an adenovirus plasmid standard using different sets of primers. The plasmid contained an insert of the human adenovirus serotype 41 hexon gene. Three sets of primers were used for amplification of the insert, producing amplicons of different lengths. Melting temperatures correlated with the lengths of amplicons. The small blue peak at around $75^{\circ} \mathrm{C}$ corresponds to the primer peak of the neHex3deg/neHex4deg system.

$6^{\circ} \mathrm{C}$. Virus stabilities showed some variation within the range of $1.0 \log _{10}$ steps after the incubation of 64 days. All serotypes could still be detected after this time period and copy numbers of specific serotypes did never decrease for more than $1.5 \log _{10}$ steps. Under environmental conditions, where temperatures and other parameters vary over time, stronger deviations may be observed. Species F adenovirus serotypes (hAdV40 and hAdV41) show high prevalence in environmental samples, but the results indicate, that other serotypes may be stable enough to allow diversity analyses up to weeks after contamination.

For such investigations, melting point analysis was adapted to adenovirus serotyping. The analyses of AdF/ AdR amplification products confirmed the calculated melting points with high accuracy. Only serotype 40 was not within the range of prediction. This may be due to genotypic variations and characterization of this specimen is ongoing. The presented investigations using adenoviruses from virus cultures suggest, that discrimination of serotypes is possible when corresponding melting points differ by more than $0.3^{\circ} \mathrm{C}$. Although only a restricted number of different serotypes could be discriminated by the primer system used, the results were consistent and seem to allow a fast classification of specimens in samples with defined matrices. Samples with a small number of serotypes may even be judged on the basis of melting curve analyses alone, using an appropriate set of melting point reference viruses. Several research groups have recently demonstrated that discrimination of norovirus, influenzavirus and fowl adenovirus species or serotypes by melting point analysis is possible [30-32]. None of these groups analyzed environmental samples, but their investigations and our current findings with human adenoviruses suggest that serotype discrimination of other virus taxa by melting point analysis is also possible in environmental samples.

Heterogeneity analyses of sewage-derived samples revealed multiple peaks or pronounced shoulders in several, but not all samples. Unambiguous sequences were obtained by direct sequencing from samples with single peak melting curves and samples with melting curves showing small shoulders, indicating that shoulders might be of artificial nature, for instance a result of insufficient purity or interfering components [33]. However, failure of heterogeneity detection by direct sequencing might simply result from concentration differences of distinct sequence products in the samples. In contrast, samples with multiple peaks could not be sequenced without cloning, suggesting heterogeneity. In complex samples, peaks may fuse and broaden, hindering correlation attempts. Therefore, we assume that, in contrast to the in vitro experiments with purified adenovirus serotypes, melting point differences of at least $1-1.5^{\circ} \mathrm{C}$ are required for the analysis of serotype heterogeneity in environmental samples.

Peaks exceeding $85^{\circ} \mathrm{C}$ could not be assigned to any accessible sequences of human or animal adenoviruses. Recent studies revealed tremendous numbers of different and as yet mostly uncharacterized species from almost every sampling site, that will employ molecular biologists as well as ecologists, taxonomists, biochemists and others for years to come [34-36]. Such high genetic diversity increases the potential risk of false positive results generated by unspecific amplification and insufficient primer design. Even if primer specificity is high, detection of new kinds of human or animal pathogenic adenoviruses is not unlikely. New animal and human adenovirus serotypes are described frequently and only limited knowledge seems to be available concerning the ecology and interspecies transmission of many serotypes [37]. Although adenoviruses were assumed to be host specific, new findings suggest that for example the feline or several primate adenoviruses are not [38-40]. It is unknown how many adenoviruses can cause zoonosis or anthroponosis, and some of them may also be amplified by the primer system used. Melting points of sequences directly depend on base composition and sequence length. The highest peak obtained from our environmental samples, measured at $86.43 \pm 0.24^{\circ} \mathrm{C}$ using the $A d F / A d R$ primer pair might belong to an unknown human or animal adenovirus with significant sequence differences to the investigated serotypes.

Revealing of unknown sequences in anthropogenic sewage by a simple method like melting point analysis 
underlines the urgent need for basic research in the field of viral biodiversity and virus ecology in particular, and genetic diversity in general. Samples hinting at unknown adenoviruses by means of melting point analyses are currently undergoing further investigation in our working group. The combined method of qPCR and melting point analyses could be used to monitor the current epidemiological status of certain virus serotypes in contaminated environmental water samples. For this assay viral nucleic acids were concentrated from $10 \mathrm{~L}$ samples, but smaller sample volumes could be suitable as well, provided that virus titers are high enough. Methods with better recovery rates will also improve the possibilities to use serotype analysis approaches.

In contrast to results using the adenovirus AdF/AdR primers, efficiencies of serotype detection varied using the Hex1deg/Hex2deg and neHex3deg/neHex4deg primer pairs. Whereas some serotypes were amplified equally well using different primer systems, others displayed variation. Longer sequences did not allow a better discrimination, although the peaks were generally sharper. In contrast, shorter sequences usually produced broader peaks. These results indicate, that careful selection of the primer system used for the PCR preceding the melting point analyses is crucial. Although exact serotyping will always need confirmation by sequencing of the amplicons, melting point analyses can provide fast information regarding the heterogeneity within a specific sample. It therefore may be a helpful tool to decide, whether further, probably costly and time-consuming investigations with regard to virus diversity, are justified.

Based on the results of melting point analyses and stability investigations, fecal adenovirus serotype 41 clearly qualified as an indicator for sewage pollution. Ad41 showed an innate stability comparable to other human pathogenic adenoviruses and was detected in all sewage derived samples. It therefore may be used for monitoring of adenovirus elimination during water treatment.

To our best knowledge, this study is the first application of melting point analyses on viruses in environmental water samples. Our results demonstrate, that several human adenovirus serotypes can be distinguished on the basis of their melting behavior. Melting point analyses of environmental samples are inexpensive, fast and accomplishable in a closed tube system, subsequent to PCR.

The described application of this method on sewagecontaminated water samples revealed its suitability for environmental samples. Although it is not required for routine monitoring, the method described in this research could be useful for epidemiological investigations.

\section{Conclusion}

Although human adenoviruses type 40 and 41 are the dominating serotypes in environmental water samples, other adenovirus serotypes may be present. Water samples obtained from processed groundwater which was artificially contaminated with $1 \%$ sewage was used to prove the principle that human adenovirus serotype diversity can be monitored using a combination of group-specific qPCR and melting curve analysis. Such melting curve analyses on PCR amplification products of environmental samples may be used as a first screening step on samples intended for further epidemiologic serotype analyses.

\section{Methods}

\section{Viruses}

Human adenovirus serotypes 1 (species C), 4 (species E), 11 (species B), 22 (species D), 31 (species A) and 40 (species F) were provided as culture supernatants by the German National Reference Laboratory for Adenoviruses, Hannover Medical School, Germany. Human adenoviruses serotypes 2 and 5 (species $C$ ) and 41 (species F) were purchased from the American type culture collection (ATCC) and cultivated in HEK 293 cells.

\section{Virus stability investigations}

For the investigation of virus stabilities, various adenovirus serotypes were diluted in processed water (ground water after iron/manganese removal) obtained from the UBA water facility in Berlin-Marienfelde. A total volume of $20 \mathrm{ml}$ was spiked with either one or multiple human adenovirus serotypes (total concentrations of $10^{5}$ viruses). The volume of $20 \mathrm{ml}$ was chosen to avoid further concentration steps for virus recovery. Virus stability was investigated over a period of eight weeks and samples were collected every four weeks. All approaches were protected from UV light and kept at either cool $\left(6 \pm 2^{\circ} \mathrm{C}\right)$ or ambient temperatures (room temperature, $20-25^{\circ} \mathrm{C}$ ), without stirring. These temperatures were chosen as representatives for cool and warm environmental conditions. $1 \mathrm{ml}$ aliquots were analyzed after nucleic acid extraction by TaqMan adenovirus consensus qPCR using the AdF/AdR primer system [27].

\section{Environmental samples}

Environmental water samples containing naturally circulating human adenoviruses were drawn during four long-time experiments, performed in fall 2009, spring and autumn 2010, and fall 2011, using the artificial stream and pond system (FSA) of the German Federal Environment Agency (UBA) in Berlin. Processed water (ground water after biological iron/manganese removal), was spiked to a final concentration of $1-5 \%(\mathrm{v} / \mathrm{v})$ sewage using primary effluent. For the present serotype analysis study, $10 \mathrm{~L}$ samples were collected at the start of these experiments and after a time-span of about one month. Sample concentration by glass wool filtration and 
flocculation was performed as described by Wyn-Jones et al. (2010) [41].

\section{Nucleic acid extraction}

Nucleic acid extraction was performed using the NucliSens Magnetic Extraction Kit (Biomérieux) according to the manufacturer's instructions. Briefly, samples were lysed in 2 volumes of lysis buffer for $10 \mathrm{~min}$ after mixing. $50 \mu \mathrm{l}$ of magnetic bead suspension were added, mixed and incubated for another 10 minutes. Magnetic beads were collected by a magnetic holder (Novagen) and washed with three different buffers, according to the manufacturer's instructions. For elution a total of $100 \mu \mathrm{l}$ elution buffer was used in two succeeding steps, but collected in one tube. Elution was carried out in a thermoshaker (Eppendorf) for $5 \mathrm{~min}$ at $60^{\circ} \mathrm{C}$ and $14000 \mathrm{rpm}$ (each step).

\section{Combination of quantitative real time PCR and melting point analyses}

For quantitative real time PCR undiluted or 1:10 diluted nucleic acid samples were measured against a standard curve of plasmid DNA containing an insert from the hexon gene sequence of adenovirus serotype 41 (pAdV41). The standard curve was performed using concentrations from $10^{1}$ to $10^{6}$ copies in 3 parallels, respectively. $10 \mu \mathrm{l}$ of diethylpyrocarbonate (DEPC)-treated, deionized water served as non-template control, while three parallels of $5 \times 10^{2}$ copies of standard were used as positive controls. Reference virus concentrations were about $10^{3}$ copies per sample. Each sample and standard, as well as positive and negative controls used during adenovirus investigations contained $12.5 \mu \mathrm{l}$ SYBR-Green Mastermix (Applied Biosystems), $0.9 \mu \mathrm{M}$ forward primer and reverse primer, respectively, and $0.5 \mu \mathrm{l}$ of DEPC treated and sterile water. Of each sample at least two parallels were measured. The primer systems used in this study are based on consensus sequences within the hexon gene region of the adenoviral genome. This conserved gene region is similar in all human and many mammalian adenovirus serotypes and encodes for the capsid hexon protein. Three different sets of forward and reverse primers were used during these investigations (see Results for further information): AdF (5'-CWT ACA TGC ACA TCK CSG G-3') and AdR (5'-CRC GGG CRA AYT GCA CCA G-3'), Hex1deg (5'GCC SCA RTG GKC WTA CAT GCA CAT C-3') and Hex2deg (5'-CAG CAC SCC ICG RAT GTC AAA-3') and neHex3deg (5'-GCC CGY GCM ACI GAI ACS TAC TTC-3') and neHex4deg (5'- CCY ACR GCC AGI GTR WAI CGM RCY AAC TA-3'). Primer systems were designed by Henroth et al. 2002 (AdF/AdR) and Allard et al. 2001 (Hex1deg/Hex2deg and neHex3deg/neHex4deg) $[27,29]$. Total volume was always $25 \mu \mathrm{l}$. Standards and primers were solved in DEPC-treated, deionized water. MicroAmp Optical 96-Well Reaction Plates (Applied
Biosystems) were used during measurement. The qPCR protocol comprised an initial hold at $95^{\circ} \mathrm{C}$ for $10 \mathrm{~min}$ (hot start), followed by 45 cycles of a 2 -step PCR protocol with a $15 \mathrm{sec}$ denaturation phase at $95^{\circ} \mathrm{C}$ and a 1 min annealing/elongation phase at $60^{\circ} \mathrm{C}$. All qPCR experiments were performed using the Applied Biosystem 7500 RT-PCR Fast system. System software version 2.0.5 was used for analyses.

DNA melting temperatures were measured directly after qPCR amplification according to the Applied Biosystem melting curve protocol. In short, after qPCR amplification the samples were heated to $95^{\circ} \mathrm{C}$ for $15 \mathrm{sec}$, cooled down to $60^{\circ} \mathrm{C}(60 \mathrm{sec})$ and subsequently heated to $95^{\circ} \mathrm{C}(30 \mathrm{sec})$ with a ramp rate of $0.5 \%$. Finally, the samples were cooled down to $60^{\circ} \mathrm{C}(15 \mathrm{sec}) . \mathrm{T}_{\mathrm{m}}$ values (mean values of several measurements) were taken from the first deviations of the melting curves. Without further software, export of the melting curves is possible, but first deviations have to be calculated manually. Our graphical data represent mean values of 2-5 melting curves. The analysis of 1:10 diluted samples derived from FSA experiments was followed by direct sequencing from the product mixture, performed by Dr. Martin Meixner GmbH, Berlin.

\section{Sequence alignments and melting point calculations}

The following human adenovirus serotypes were used for sequence alignments, NCBI GenBank or RefSeq accession numbers are listed in square brackets: hAdVA serotypes: 12 [X73487.1], 18 [DQ149610.1] and 31 [DQ149611.1]; hAdVB serotypes: 3 [DQ086466.1], 7 [AF515814.1], 11 [AB330092.1], 14 [DQ149612.1], 16 [X74662.1], 21 [AY008279.1], 34 [AB052911.1], 35 [AB052912.2], 50 [DQ149643.1] and 55 [FJ643676.1]; hAdVC serotypes: 1 [AF534906.1], 2 [J01917.1], 5 [AC_000008.1] and 6 [DQ149613.1]; hAdVD serotypes: 8 [DQ149614.1], 9 [AJ854486.1], 10 [DQ149615.1], 13 [DQ149616.1], 15 [DQ149617.1], 17 [AF108105.1], 19 [DQ149618.1], 20 [DQ149619.1], 22 [DQ149620.1], 23 [DQ149621.1], 24 [DQ149622.1], 25 [DQ149623.1], 26 [DQ149624.1], 27 [DQ149625.1], 28 [DQ149626.1], 29 [DQ149627.1], 30 [DQ149628.1], 32 [DQ149629.1], 33 [DQ149630.1], 36 [DQ149631.1], 37 [DQ149632.1], 38 [DQ149633.1], 39 [DQ149634.1], 42 [DQ149635.1], 43 [DQ149636.1], 44 [DQ149637.1], 45 [DQ149638.1], 46 [AY875648.1], 47 [DQ149640.1], 48 [EF153473.1], 49 [DQ393829.1], 51 [DQ149642.1], 53 [FJ169625.1], 54 [AB448770.2] and 56 [HM770721.2]; hAdVE serotypes: 4 [AY487947.1]; hAdVF serotypes: 40 [AB330121.1] and 41 [AB330122.1]; hAdVG serotypes: 52 [DQ923122.2]. Non-human adenoviruses used in the analysis were the bovine adenovirus A (bAdVA) [NC_006324.1], canine adenovirus serotypes 1 (cAdV1) [AC_000003.1] and 2 (cAdV2) [DQ839392.1], the feline adenovirus (fAdV) [AY512566.1], the murine adenovirus serotype 2 (mAdV2) 
[NC_014899.1], the ovine adenovirus A (oAdVA) [NC_002513.1] and the porcine adenovirus C (pAdVC) [NC_002702.1]. Melting point calculations were using the OligoCalc program with parameters of $900 \mathrm{~nm}$ primer and $50 \mathrm{mM}$ salt concentrations [28].

\section{Competing interests}

The authors declare that they have no competing interests.

\section{Authors' contributions}

$\mathrm{NMH}$ conceived this study, designed and coordinated the experiments, participated in all experiments performed, analyzed and interpreted the data and wrote the manuscript. MD performed several important experiments, including sample preparation and adenovirus serotyping from sewagederived samples by restriction fragment analyses. HCS co-designed the study, participated in data analysis and revision of the manuscript. RS participated in interpreting the data and revised the manuscript. All authors read and approved the final manuscript.

\section{Acknowledgements}

The authors thank their colleagues in FG II1.4 and II1.5 for technical support and A. Heim (German National Reference Laboratory for Adenoviruses, Hannover Medical School, Germany) for providing reference viruses. This research was funded by the German Federal Environment Agency and a grant from the Deutsche Forschungsgemeinschaft (DFG, SE 508/2-1) to HCS for investigation of viruses in water samples. $\mathrm{NMH}$ was supported by the grant during his Ph.D. thesis. The funding agencies did not have any influence on this publication and the research presented herein represents the oppinions of the authors, but not necessarily the oppinions of the funding agencies.

Received: 26 September 2012 Accepted: 17 May 2013 Published: 10 June 2013

\section{References}

1. Longe EO, Ogundipe AO: Assessment of wastewater discharge impact from a sewage treatment plant on lagoon later, Lagos, Nigeria. Res J Appl Sci Eng Technol 2010, 2(3):274-282.

2. Hrudey SE, Hrudey EJ: Published case studies of waterborne disease outbreaks - evidence of a recurrent threat. Water Environ Res 2007, 79(3):233-245

3. Coelho SM, Rijstenbil JW, Brown MT: Impacts of anthropogenic stresses on the early development stages of seaweeds. J Aquat Ecosyst Stress Recovery 2000, 7(4):317-333.

4. Jobling $S$, Nolan M, Tyler CR, Brighty G, Sumpter JP: Widespread sexual disruption in wild fish. Environ Sci Technol 1998, 32(17):2498-2506.

5. Calabrese A: How some pollutants affect embryos and larvae of american oyster and hard-shell clam. Mar Fish Rev 1972, 34(11-12):66-77.

6. Khan FA, Ansari AA: Eutrophication: An ecological vision. Bot Rev 2005, 71(4):449-482.

7. Smith SV: Responses of Kaneohe Bay, Hawaii, to relaxation of sewage stress. In Estuaries and Nutrients. Edited by Neilson BJ, Cronin LE. Clifton, New Jersey: Humana Press Inc; 1981:391-410.

8. Appelbee AJ, Thompson RCA, Measures LM, Olson ME: Giardia and Cryptosporidium in harp and hooded seals from the Gulf of St. Lawrence, Canada. Vet Parasit 2010, 173(1-2):19-23.

9. WHO: Health Based Monitoring of Recreational Waters: The Feasibility of a New Approach (the 'Annapolis Protocol'). Geneva: World Health Organisation; 1999.

10. Enriquez $C E$, Hurst $C J$, Gerba CP: Survival of the enteric adenoviruses 40 and 41 in tap, sea, and waste water. Water Res 1995, 29(11):2548-2553.

11. Medema GJ, Shaw S, Waite M, Snozzi M, Morreau A, Grabow W: Catchment characterisation and source water quality. In Assessing microbial safety of drinking water: Improving approaches and methods. Edited by Dufour A, Snozzi M, Köster W, Bartram J, Ronchi E, Fewtrell L. London, UK: IWA Publishing, on behalf of the World Health Organization and the Organisation for Economic Co-operation and Development; 2003:111-158.

12. Bosch A: Human enteric viruses in the water environment: a minireview. Internatl Microbiol 1998, 1(3):191-196.
13. Leclerc H, Schwartzbrod L, Dei-Cas E: Microbial agents associated with waterborne diseases. In Microbial waterborne pathogens. Edited by Cloete TE, Rose J, Nel LH, Ford T. London, UK: IWA Publishing London; 2004:1-54.

14. Ashbolt NJ, Grabow WOK, Snozzi M: Indicators of microbial water quality. In Water quality: Guidelines, standards and health. Edited by Fewtrell L, Barthram J. London, UK: IWA Publishing; 2001:289-316.

15. Figueras $\mathrm{MJ}$, Borrego JJ: New perspectives in monitoring drinking water microbial quality. Int J Environ Res Public Health 2010, 7(12):4179-4202.

16. US EPA: Drinking water contaminant list 3 - Final. Fed Reg 2009, 74(194):51850-51862.

17. WHO: Guidelines for Drinking-water Quality. Volume 1. 3rd edition. Geneva: World Health Organization; 2008.

18. Fong T-T, Lipp EK: Enteric viruses of humans and animals in aquatic environments: Health risks, detection, and potential water quality assessment tools. Microbiol Mol Biol Rev 2005, 69(2):357-371.

19. Beuret C: Simultaneous detection of enteric viruses by multiplex realtime RT-PCR. J Virol Meth 2004, 11(1):1-8.

20. Aslan A, Xagoraraki I, Simmons FJ, Rose JB, Dorevitch S: Occurrence of adenovirus and other enteric viruses in limited-contact freshwater recreational areas and bathing waters. J Appl Microbiol 2011, 111(5):1250-1261.

21. Siqueira-Silva J, Perez Yeda F, Favier A-L, Mezin P, Silva ML, Médici Barrella K, Mehnert DU, Fender P, Hársi CM: Infection kinetics of human adenovirus serotype 41 in HEK 293 cells. Mem Inst Oswaldo Cruz 2009, 104(5):736-744.

22. Jiang SC, Han J, He J-W, Chu W: Evaluation of four cell lines for assay of infectious adenoviruses in water samples. J Water Health 2009, 7(4):650-656.

23. Xu W, McDonough MC, Erdman DD: Species-specific identification of human adenoviruses by a multiplex PCR assay. J Clin Microbiol 2000, 38(11):4114-4120.

24. Balkin HB, Margolin AB: Detection of poliovirus by ICC/qPCR in concentrated water samples has a greater sensitivity and is less costly using BGM cells in suspension as compared to monolaysers. Virology J 2010, 7:282-285

25. Murugesan G, Hsi ED: Development of a clinical assay for JAK2 V617F genotyping in chronic myeloproliferative disorders. Pathol Res 2006:1-4.

26. Reed $\mathrm{GH}$, Wittwer $\mathrm{CT}$ : Sensitivity and specificity of single-nucleotide polymorphism scanning by high-resolution melting analysis. Clin Chem 2004, 50(10):1748-1754.

27. Hernroth BE, Conden-Hansson A, Rehnstam-Holm A, Girones R, Allard AK: Environmental factors influencing human viral pathogens and their potential indicator organisms in the blue mussel, Mytilu eduli: the first Scandinavian report. Appl Environ Microbiol 2002, 68(9):4523-4533.

28. OligoCalc: Oligonucleotide properties calculator. [http://www.basic. northwestern.edu/biotools/oligocalc.html]

29. Allard A, Albinsson B, Wadell G: Rapid typing of human adenoviruses by a general PCR combined with restriction endonuclease analysis. $J$ Clin Microbiol 2001, 39(2):498-505.

30. Scipioni A, Mauroy A, Ziant D, Saegerman C, Thiry E: A SYBR green RT-PCR assay in single tube to detect human and bovine noroviruses and control for inhibition. J Virol Meth 2008, 5(94):1-8.

31. Lin J-H, Tseng C-P, Chen Y-J, Lin C-Y, Chang S-S, Wu H-S, Cheng J-C: Rapid differentiation of influenza A virus subtypes and genetic screening for virus variants by high-resolution melting analysis. J Clin Microbiol 2008, 46(3):1090-1097.

32. Steer PA, Kirkpatrick NC, O'Rourke D, Noormohammadi AH: Classification of fowl adenovirus serotypes using high resolution melting curve analysis of the hexon gene region. J Clin Microbiol 2009, 47(2):311-321.

33. D'haene B, Hellemans J: The importance of quality control during $\mathrm{qPCR}$ data analysis. Int Drug Discov 2010:18-24.

34. Mclnerney JO, Mullarkey M, Wernecke ME, Powell R: Bacteria and Archaea: Molecular techniques reveal astonishing diversity. Biodiversity 2002, 3(2):3-10.

35. Breitbart M, Thompson LR, Suttle CA, Sullivan MB: Exploring the vast diversity of marine viruses. Oceanography 2007, 20(2):135-139.

36. Cantalupo PG, Calgua B, Zhao G, Hundesa A, Wier AD, Katz JP, Grabe M, Hendrix RW, Girones R, Wang D, Pipas JM: Raw sewage harbors diverse viral populations. mBio 2011, 2(5):e00180-11.

37. Maluquer de Motes C, Hundesa A, Almeida FC, Bofill-Mas S, Girones R: Isolation of a novel monkey adenovirus reveals a new phylogenetic clade in the evolutionary history of simian adenoviruses. Virology $J 2011$ 8:125-132. 
38. Phan TG, Shimizu H, Nishimura S, Okitsu S, Maneekarn N, Ushijima H: Human adenovirus type 1 related to feline adenovirus: evidence of interspecies transmission. Clin Lab 2006, 52(9-10):515-518.

39. Chen EC, Yagi S, Kelly KR, Mendoza SP, Maninger N, Rosenthal A, Spinner A, Bales KL, Schnurr DP, Lerche NW, Chiu CY: Cross-species transmission of a novel adenovirus associated with a fulminant pneumonia outbreak in a New World Monkey colony. PLOS Pathog 2011, 7(7):e1002155.

40. Wevers D, Metzger S, Babweteera F, Bieberbach M, Boesch C, Cameron K, Couacy-Hymann E, Cranfield M, Gray M, Harris LA, Head J, Jeffery K, Knauf S, Lankester F, Leendertz SAJ, Lonsdorf E, Mugisha L, Nitsche A, Reed P,

Robbins M, Travis DA, Zommers Z, Leendertz FH, Ehlers B: Novel adenoviruses in wild primates: a high level of genetic diversity and evidence of zoonotic transmissions. J Virol 2001, 85(20):10774-10784

41. Wyn-Jones AP, Carducci A, Cook N, D’Agostino MD, Divizia M, Fleischer J, Gantzer C, Gawler A, Girones R, Höller C, de Roda Husman AM, Kay D, Kozyra I, López-Pila J, Muscillo M, São José Nascimento M, Papageorgiou G, Rutjes S, Sellwood J, Szewzyk R, Wyer M: Surveillance of adenoviruses and noroviruses in European recreational waters. Water Res 2011, 45(3):1025-1038.

doi:10.1186/1743-422X-10-190

Cite this article as: Hartmann et al:: Monitoring of adenovirus serotypes in environmental samples by combined PCR and melting point analyses. Virology Journal 2013 10:190.

\section{Submit your next manuscript to BioMed Central and take full advantage of:}

- Convenient online submission

- Thorough peer review

- No space constraints or color figure charges

- Immediate publication on acceptance

- Inclusion in PubMed, CAS, Scopus and Google Scholar

- Research which is freely available for redistribution 